\title{
EGFR inhibitors sensitize non-small cell lung cancer cells to TRAIL-induced apoptosis
}

\author{
Fei $\mathrm{Xu}^{1,2}$, Ying Tian ${ }^{1,2}$, Yan Huang ${ }^{1,2}$, Ling-Ling Zhang ${ }^{1,2}$, Zheng-Zheng Guo ${ }^{1,2}$, \\ Jia-Jia Huang ${ }^{1,2}$ and Tong-Yu Lin ${ }^{1,2}$
}

\section{Abstract}

Apoptosis induced by tumor necrosis factor-related apoptosis-inducing ligand (TRAIL) can be regulated by the epidermal growth factor (EGF) signaling pathway. In this study, recombinant adenoviral vectors that encode TRAIL gene from the hTERT/RGD promoter (AdTRAIL) was combined with drugs including gefitinib, elotinib, and cetuximab that inhibit EGFR and the EGF signaling pathway in non-small cell lung cancer (NSCLC) cell lines to investigate their antitumor activity. In vitro, compared to single reagent, AdTRAIL combined with EGFR inhibitors reduced proliferation and enhanced apoptosis in H460, A549, and SW1573 cell lines. Western blot results suggested that these effects were relative to upregulation of pro-apoptosis protein BAX and down-regulation of $\mathrm{p}$-AKT. In vivo, AdTRAIL combined with cetuximab resulted in a significant growth reduction in $\mathrm{H} 460$ xenografts without damage to the main organs of nude mice. Histological examination and TUNEL analyses of xenografts showed that cetuximab enhanced cell apoptosis induced by AdTRAIL. These results indicate that EGFR inhibitors enhanced AdTRAIL anti-tumor activity in NSCLC cell lines and that inhibiting the AKT pathway played an important role in this enhancement.

Key words Tumor necrosis factor-related apoptosis-inducing ligand (TRAIL), epidermal growth factor receptor (EGFR), gene therapy, target therapy, non-small cell lung cancer

Non-small cell lung cancer (NSCLC) is the leading cause of death from cancer throughout the world. Despite the improvements in chemotherapy and radiotherapy, long-term survival of patients with NSCLC still remains low. For this reason, novel and more effective treatments and strategies for NSCLC are critically needed.

Epidermal growth factor receptor (EGFR) is overexpressed in $32 \%$ to $81 \%$ of NSCLCs and affects all aspects of carcinogenesis, including cell growth,

\footnotetext{
Authors' Affllatlons: 'State Key Laboratory of Oncology in South China, Guangzhou, Guangdong 510060, P. R. China; 'Department of Medical Oncology, Sun Yat-sen University Cancer Center, Guangzhou, Guangdong 510060, P. R. China.

Corresponding Author: Tong-Yu Lin, Department of Medical Oncology, Sun Yat-sen University Cancer Center, Guangzhou, Guangdong 510060, P. R. China; Email: lintongyu@sysucc.org.cn; Tel: +86-20-87343366. dol: $10.5732 /$ cjc.011.10107
}

invasion, angiogenesis, and metastasis ${ }^{[1-4]}$. Thus, EGFR inhibitors are a major focus in NSCLC therapies. Gefitinib (ZD1839, Iressa) and erlotinib (OSI-774, Tarceva) are small molecule tyrosine kinase inhibitors (TKIs) that inhibit the internal catalytic activity of EGFR and decrease lung cancer cell proliferation. In 2005, both gefitinib and erlotinib are approved by the Food and Drug Administration (United States) as single agent therapy for NSCLC following chemotherapy failure. Cetuximab is an immunoglobulin ( $\mathrm{lg}) \mathrm{G} 1$ monoclonal antibody that binds to the extracellular domain of EGFR, effectively blocking the ligand-receptor interaction and promoting receptor internalization. Recent studies have shown that combining cetuximab with first-line chemotherapy improves treatment efficacy in patients with advanced NSCLC ${ }^{[5-7]}$. However, in clinical practice, EGFR inhibitors have produced objective responses in only a small fraction $(9 \%$ to $30 \%)$ of patients with advanced NSCLC ${ }^{[8-10]}$. Thus, strategies having synergism with EGFR-targeting drugs are undoubtedly needed. The combination of 
EGFR TKIs and standard chemotherapy has not been successful to this end ${ }^{[11-14]}$; however, novel therapies that have this synergetic effect are still expected.

Tumor necrosis factor-related apoptosis-inducing ligand (TRAIL, also known as Apo-2L) ${ }^{[15]}$ is a member of the tumor necrosis factor (TNF) family. Identified in 1995, TRAIL is a type II membrane protein that can be cleaved from the cell surface to form a soluble ligand that induces apoptosis in a wide variety of cancer cell lines ${ }^{[16]}$, including NSCLC cells, without inducing significant toxicity in most normal cells ${ }^{[17-19]}$. TRAIL-expressing adenoviral vectors, such as AdTRAIL, can kill tumor cells directly and stimulate a potent bystander effect through the presentation of TRAIL by transduced normal cells ${ }^{[20-22]}$. Studies show that the antitumor effects of different TRAIL therapies, including AdTRAIL, are comparable in NSCLC cells ${ }^{[23]}$. Furthermore, some studies have demonstrated that apoptosis induced by TRAIL could be modulated by cellular levels of $\mathrm{AKT}^{[24]}$, an active member of the EGF pathway.

We hypothesized that EGFR inhibitors have synergism with AdTRAIL and that inhibiting survival signaling pathways, e.g. by using EGFR inhibitors, would enhance the antitumor activity of apoptosis signaling pathway activators, such as AdTRAIL. In this study, we preliminarily evaluated the synergy between EGFR pathway inhibitors and AdTRAIL, and our observations demonstrated that EGFR inhibitors, such as TKIs and cetuximab, could enhance AdTRAIL activity in NSCLC.

\section{Materials and Methods}

\section{Cell lines and reagents}

Lung large cell cancer cell line $\mathrm{H} 460$, lung alveolar cell carcinoma cell line SW1573, and lung adenocarcinoma cell line A549 were provided by the State Key Laboratory of Oncology in South China. H460 and $\mathrm{A} 549$ cells were cultured at $37^{\circ} \mathrm{C}$ in a humidified incubator in an atmosphere containing $5 \% \quad \mathrm{CO}_{2}$ and grown in RPMI-1640 media supplemented with 10\% heat-inactivated fetal bovine serum, antibiotics, and glutamine. SW1573 cells were cultured in DMEM tissue culture medium (Cambrex Bioscience). The EGFR inhibitors gefitinib (IRESSA, AstraZeneca Co.), elotinib (TARCEVA, Roche Co.), and cetuximab (ERBITUX, Merck Co.) were supplied by the Department of Pharmacy of Sun Yat-sen University Cancer Center. Adenoviral vectors that contained TRAIL gene from the hTERT/RGD promoter (AdTRAIL) or no transgene (AdG) were constructed as described previously ${ }^{[25}$. The expansion, purification, titration, and quality analysis of the vectors were performed as described previously ${ }^{[21,25]}$. The adenoviral titer was determined by the absorbency of the dissociated virus at a wavelength of $260 \mathrm{~nm}$ (one $A_{260}$ unit $=1012$ viral particles $/ \mathrm{mL}$ ), whereas titers that were determined by plaque assay were used for supplementary data. The infectious units (ifu) for the virus were determined using the Adeno- $X$ rapid titer kit (Clontech, USA) according to the manufacturer's protocol.

\section{Cell viability analysis}

Cells were seeded at a density of $1 \times 10^{4}$ cells in $100 \mu \mathrm{L}$ of culture medium/well into flat-bottomed 96-well plates, treated with indicated concentration of EGFR inhibitors, AdTRAIL, or combined treatment, and incubated for $72 \mathrm{~h}$ at $37^{\circ} \mathrm{C}$ in a humidified atmosphere containing $5 \% \mathrm{CO}_{2}$. Cell viability was determined with the 3(4,5-dimethylthiazol-2-yl)-2,5-diphenyltetrazolium bromide (MTT) assay. The absorbency was recorded at $570 \mathrm{~nm}$ using a spectrophotometer (Bio-Rad, USA). Each experiment was performed in quadruplicate and repeated at least three times.

\section{Cell cycle analysis}

Cells were treated with indicated combinations for $72 \mathrm{~h}$, washed twice with cold PBS, and fixed with $70 \%$ ethanol for $30 \mathrm{~min}$ at $4^{\circ} \mathrm{C}$. After incubation with RNase $\mathrm{A}$ (1 $\mathrm{mg} / \mathrm{mL}$ in PBS) for $30 \mathrm{~min}$ at $37^{\circ} \mathrm{C}$, cells were stained with $50 \mathrm{mg} / \mathrm{mL}$ of propidium iodide (Sigma, USA) for 15 min before being analyzed on a FACScalibur using Cell Quest software (Beckton Dickinson, USA). The sub- $\mathrm{G}_{1}$ population was calculated to estimate the apoptotic cell population.

\section{Annexin V analysis}

Cells were treated with indicated combinations for $72 \mathrm{~h}$, and apoptosis was analyzed with FITC-annexin V staining (Sigma, USA) according to the manufacturer's instructions. Cells were washed twice with cold PBS and then resuspended in $1 \times$ binding buffer at a concentration of $1 \times 10^{6}$ cells $/ \mathrm{mL}$. The cell suspension was placed to 5 -mL culture tubes ( $100 \mu \mathrm{L} /$ tube), stained with Annexin $\mathrm{V}$ and Vital Dye, then incubated at room temperature in the dark for $15 \mathrm{~min}$, and finally added with $1 \times$ binding buffer (400 $\mu \mathrm{L} /$ tube). Cell apoptosis was detected by flow cytometry within $15 \mathrm{~min}$.

\section{Antibodies and Western blot analysis}

The following antibodies were used: anti-phosphoEGFR and anti-phospho-Akt (Cell Signaling Technology, Inc., USA); anti-phospho-MAP kinase 1/2 (Erk 1/2) (Upstate, USA); and caspase-3, BAX, NF-kB, and 
anti- $\beta$-actin (Sigma, USA). Cells were treated as indicated for $36 \mathrm{~h}$ and then lysed in cold lysis buffer containing $1 \%$ Triton $\mathrm{X}-100$ and phosphatase inhibitors. Samples were reduced and gel electrophoresis was performed using NuPage Bis-Tris $4 \%$ to $12 \%$ gels. After electrophoresis, the proteins were transferred onto nitrocellulose membranes and the membranes were blocked for $2 \mathrm{~h}$ at room temperature. After rinsing with TBS buffer containing $0.05 \%$ Tween20 3 times, each time $10 \mathrm{~min}$, the membranes were incubated sequentially with indicated antibody and secondary antibody. After $2 \mathrm{~h}$ of incubation at $37^{\circ} \mathrm{C}$, the membranes were rinsed and the images were developed by ECL. The results were analyzed by SensiAnsys gel document and analysis systems.

\section{Animal experiments}

Animal studies were carried out in accordance with the Guidelines for the Care and Use of Laboratory Animals (NIH Publication number 85-23) and the institutional guidelines of Sun Yat-sen University Cancer Center. Human $\mathrm{H} 460$ lung cancer xenografts were established in female nude mice (4-5 weeks old) by subcutaneous inoculation of tumor tissue $(2 \mathrm{~mm}$ in diameter) into the dorsal flank of each mouse. Nine days later, mice were randomized into 4 groups, and the tumor volume in different groups was balanced. Then, mice underwent injection of PBS, AdTRAIL, cetuximab, or AdTRAIL in combination with cetuximab. Adenoviral vectors were injected into tumors at $8 \times 10^{8}$ ifu/tumor once every $5 \mathrm{~d}$ for a total of 5 injections. Cetuximab was injected into the abdominal cavity at $0.5 \mathrm{mg} / \mathrm{mouse}$ once every $3 \mathrm{~d}$ for a total of 8 injections. Mouse weight and tumor size were measured twice per week. The tumor volume was calculated as follows: $3.1416 / 6 \times(a+b)^{3 / 8}$, in which " $a$ " denotes the longest diameter and " $b$ " is the shortest diameter of tumor. Mice were killed on the 29th day after the first treatment. Tumor inhibition rate was calculated as follows: (mean tumor weight of control group - mean tumor weight of treatment group) / mean tumor weight of control group $\times 100 \%$.

\section{Pathologic examination}

Tissues were fixed in formalin and embedded in paraffin. Sections were made at 3-mm thick, stained with hematoxylin \& eosin (HE), and observed under a microscope.

\section{TUNEL assay}

Apoptosis in tumor tissue was assessed with Dead End Colorimetric TUNEL (terminal deoxynucleotidyl transferase-mediated $\quad 2^{\prime}$-deoxyuridine $\quad 5^{\prime}$-triphosphate
[dUTP] nick-end labeling) System (Roche) according to the manufacturer's instructions. The paraffin-embedded sections were de-waxed and then digested with proteinase $\mathrm{K}$ for $20 \mathrm{~min}$ and $\mathrm{H}_{2} \mathrm{O}_{2}$ for $5 \mathrm{~min}$ at room temperature. After washing with PBS, sections were labeled with TUNEL reaction mixture, stained with diaminobenziol (DAB) for $10 \mathrm{~min}$, and then washed with PBS. Apoptosis was detected under a fluorescent microscope with FITC staining.

\section{Statistical analysis}

All data are presented as mean \pm standard deviation (SD). Intergroup differences were determined using the unpaired Student's $t$ test. A value of $P<0.05$ was considered significant. All results were obtained from at least three independent experiments. Differences between the treatment groups were assessed by ANOVA using the statistical software SPSS11.0.

\section{Results}

NSCLC cell lines had varying sensitivities to AdTRAIL or EGF inhibitor monotherapy

Before testing the combined effect of AdTRAIL gene therapy and EGF pathway-targeting therapy, we first evaluated the cytotoxicity of AdTRAIL or EGF inhibitor monotherapy in NSCLC cell lines H460, SW1573, and A549. Our data showed a dose-dependent cytotoxic effect of AdTRAIL at 30 to $300 \mathrm{MOI}$ (multiplicity of infection) in these NSCLC cell lines (Figure 1A). The antitumor effect of AdTRAIL was significant at $100 \mathrm{MOI}$ and higher. With regard to EGFR inhibitor therapy, our study showed that $\mathrm{IC}_{50}$ values of both gefinitib and erlotinib in NSCLC cell lines exceeded $5.0 \mu \mathrm{mol} / \mathrm{L}$ (Figure 1B), which suggested that these NSCLC cell lines had mild response to TKIs. Cetuximab at $250 \mu \mathrm{g} / \mathrm{L}$ had varying and mild cytotoxicity on NSCLC cell lines (Figure 1C). These results indicated that these NSCLC cell lines had low sensitivity or were resistant to EGF inhibitor monotherapy.

\section{AdTRAIL in combination with EGFR inhibitors reduced NSCLC cell viability}

H460, SW1573, and A549 cells were treated with AdTRAIL or AdG at an MOI of 50 , at which the effect of AdTRAIL was modest, combined with varying concentrations of gefinitib, elotinib, or cetuximab. The antitumor activity of AdTRAIL was increased when AdTRAIL was combined with EGFR inhibitors (Figure 2). Moreover, the antitumor activity of combined treatment increased proportionally with increasing doses of the 


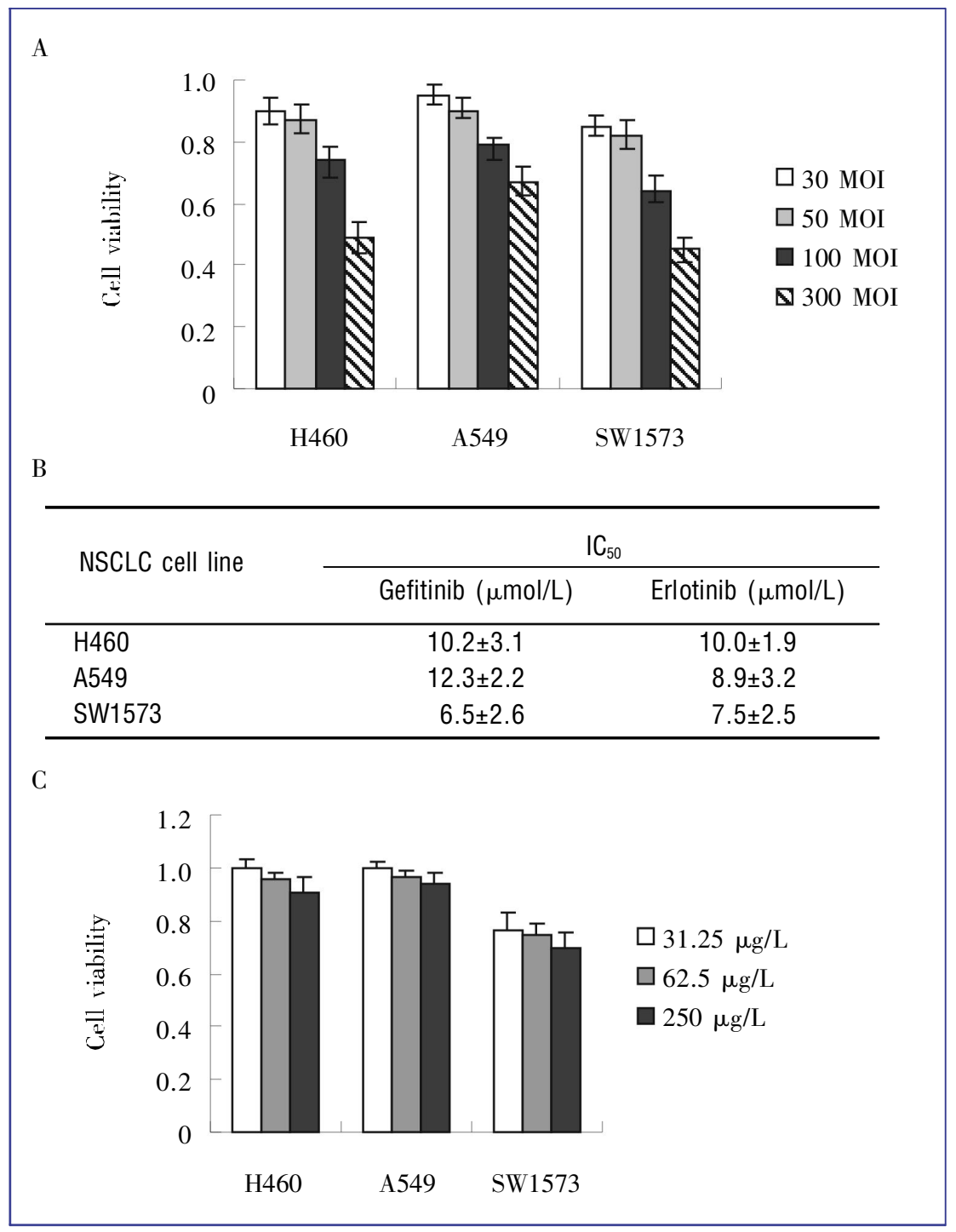

Figure 1. Non-small cell lung cancer (NSCLC) cell lines H460, SW1573, and A549 show varying sensitivities to tumor necrosis factor-related apoptosisinducing ligand (TRAIL) or epidermal growth factor receptor (EGFR) inhibitor monotherapy. A, cell viability determined by the MTT assay on the third day after treatment with AdTRAIL at 30 to 300 MOI (multiplicity of infection). The cellkilling effect of AdTRAIL was dosedependent in these NSCLC cell lines. B, the $50 \%$ inhibition concentration $\left(I_{50}\right)$ of gefitinib and elotinib in these NSCLC cell lines. C, cell viability determined by the MTT assay after a single administration of cetuximab. Slight and negligible growth inhibition was observed in the $\mathrm{H} 460$ and A549 cell lines. All data are presented as mean \pm standard deviation (SD) of three experiments.

EGFR inhibitors. These results suggested that EGFR inhibitors could enhance the antitumor effect of AdTRAIL in NSCLC cell lines.

\section{EGFR inhibitors enhanced cell apoptosis induced by AdTRAIL}

In order to quantitatively measure cell apoptosis, the population of $\mathrm{H} 460$ cells with sub-G $\mathrm{G}_{1}$ DNA content was determined using flow cytometry. Combined treatment with EGFR inhibitors and AdTRAIL resulted in significantly increased cells with sub-G ${ }_{1}$ DNA content compared to treatment with EGFR inhibitors or AdTRAIL alone (Figure $3 \mathrm{~A}$ ). The results of annexin $\mathrm{V}$ staining also showed that apoptosis increased by combination treatment than by AdTRAIL alone (Figure 3B). These findings confirmed that EGFR inhibitors enhanced apoptosis induced by AdTRAIL.

\section{Combined treatment up-regulated BAX activity and down-regulated p-AKT level}

The pro-apoptotic protein BAX plays a pivotal role in apoptosis induced by TRAIL. As shown in Figure 4A, combined treatment with $100 \mathrm{MOI}$ AdTRAIL and EGFR inhibitors up-regulated apoptotic BAX levels significantly. These results confirmed that EGFR inhibitors enhanced AdTRAIL-induced apoptosis. In this experiment, downstream active members of EGFR pathway, including p-EGFR, p-AKT, p-ERK, and NF-kB, were analyzed by Western blotting after $36 \mathrm{~h}$ of treatment. As shown in Figure 4B, p-AKT levels decreased after $36 \mathrm{~h}$ of combined treatment compared to control and single agent treatment. In contrast, $p$-EGFR levels remained stable or decreased slightly after combined treatment with AdTRAIL and cetuximab. $\mathrm{p}-\mathrm{ERK}$ and NF-kB were 


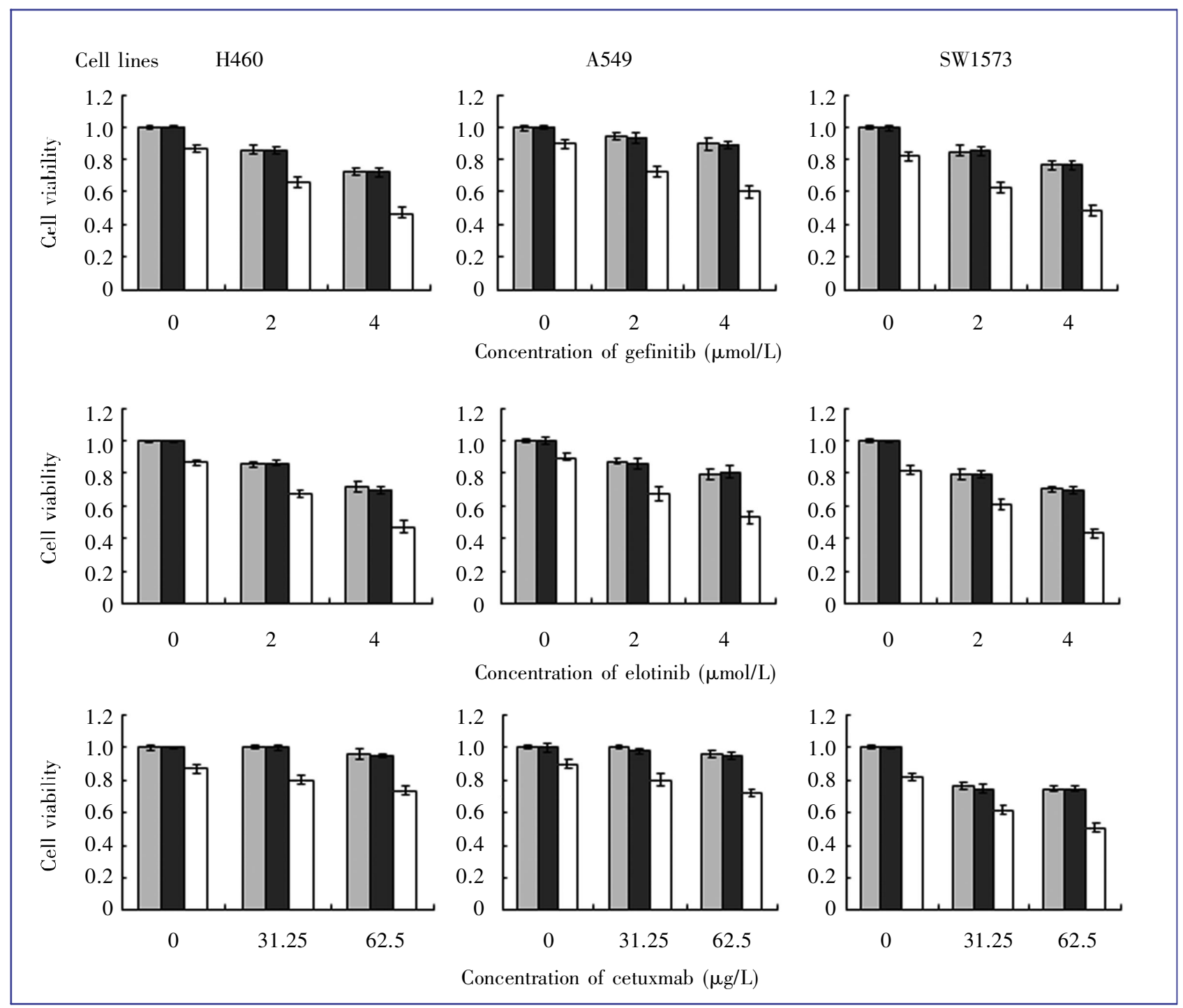

Figure 2. EGFR inhibitors and AdTRAIL inhibit NSCLC cell viability. The cells were treated with indicated treatments, including $50 \mathrm{MOI}$ of AdTRAIL or adenoviral vectors that contained CMV (AdG) combined with varying concentrations of gefinitib, elotinib, or cetuximab for $72 \mathrm{~h}$. Cell viability was determined by MTT assays. All data are presented as mean \pm SD of three experiments. Results show that EGFR inhibitors could enhance the antitumor effects of AdTRAIL in NSCLC cells. ( $\square$ EGFR inhibitors; $\square$ EGFR inhibitors plus AdG; $\square$ EGFR inhibitors plus AdTRAIL)

not down-regulated any more by combined treatment than by AdTRAIL alone. Thus, down-regulation of $p-A K T$ was associated with apoptosis enhancement, suggesting that this signaling pathway may play a role in the synergism between AdTRAIL and EGFR inhibitors.

\section{Suppression of tumor growth by AdTRAIL combined with cetuximab in vivo}

To test whether EGFR inhibitors enhance the apoptosis induced by AdTRAIL in vivo, we treated $\mathrm{H} 460$ xenograft-bearing female nude mice with PBS, cetuximab alone, AdTRAIL alone, or AdTRAIL in combination with cetuximab and monitored tumor growth for 29 days after treatment. The results revealed substantial tumor growth reduction after treatment with AdTRAIL alone or in combination with cetuximab (Figure $5 A$ ) but not after combination with cetuximab alone or PBS. Furthermore, compared to AdTRAIL alone, AdTRAIL combined with cetuximab significantly inhibited tumor growth $(P<0.05)$. Thus, when used alone, cetuximab had no effect on $\mathrm{H} 460$ xenografts, but when combined with AdTRAIL, cetuximab enhanced the antitumor activity of AdTRAIL. On the 29th day after treatment, mice were killed and xenograft tumors were weighed (Figure 5B). The mean tumor weights were $(1.72 \pm 0.40) \mathrm{g}$ in PBS control group $(n=8), \quad(1.52 \pm$ $0.72) \mathrm{g}$ in cetuximab group $(n=8),(1.25 \pm 0.48) \mathrm{g}$ in 


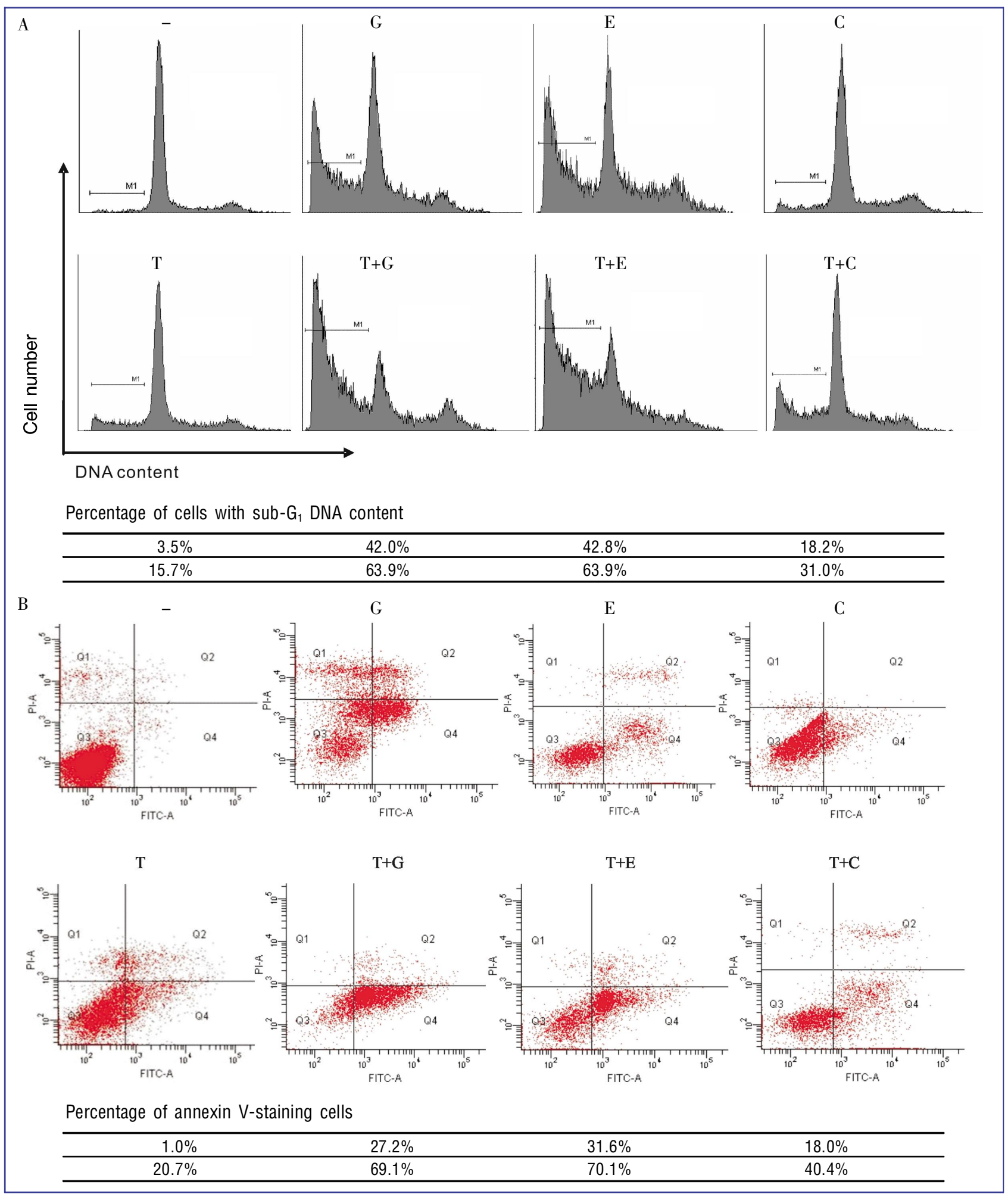

Figure 3. EGFR inhibitors enhance apoptosis of $\mathbf{H} 460$ cells induced by AdTRAIL. H460 cells were treated with the indicated concentrations of AdTRAIL (T, AdTRAIL $100 \mathrm{MOl}$ ), or EGFR inhibitors (G, gefitinib $8 \mu \mathrm{mol} / \mathrm{L}$; E, elotinib $8 \mu \mathrm{mol} / \mathrm{L}$; C, cetuximab $100 \mu \mathrm{g} / \mathrm{L}$ ), or both for $48 \mathrm{~h}$. A, DNA content was analyzed by flow cytometry. Combination treatment with AdTRAIL and EGFR inhibitors resulted in increased sub-G $\mathrm{G}_{1}$ DNA content in cells compared to treatment with AdTRAIL or EGFR inhibitors alone. B, flow cytometry analysis of apoptotic cells was performed using annexin V-FITC. The percentage of annexin V-staining cells increased with combination treatment. 


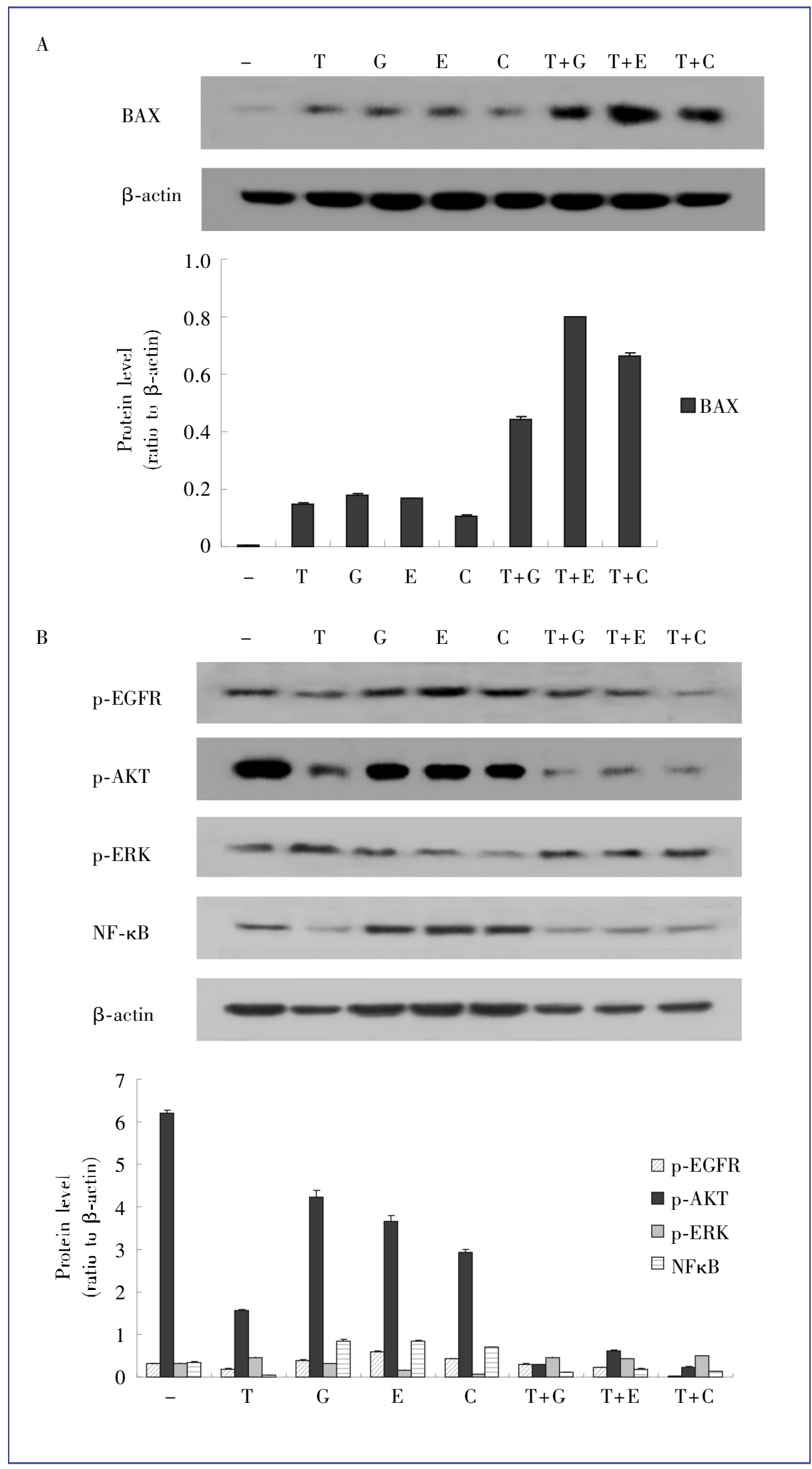

Figure 4. H460 cells were treated with indicated concentrations of agents ( $T$, AdTRAIL 100 MOI; G, gefitinib 2 $\mu \mathrm{mol} / \mathrm{L} ; \quad \mathrm{E}$, elotinib 2 $\mu \mathrm{mol} / \mathrm{L} ; \quad C$, cetuximab $62.5 \mu \mathrm{g} / \mathrm{L})$ for $36 \mathrm{~h}$. A representative study is shown, and two additional experiments yielded similar results. A, the expression of apoptotic protein Bax was up-regulated in H460 cells significantly after combination treatment. $B$, the levels of $p$-AKT in $\mathrm{H} 460$ cells decreased after combination treatment. 


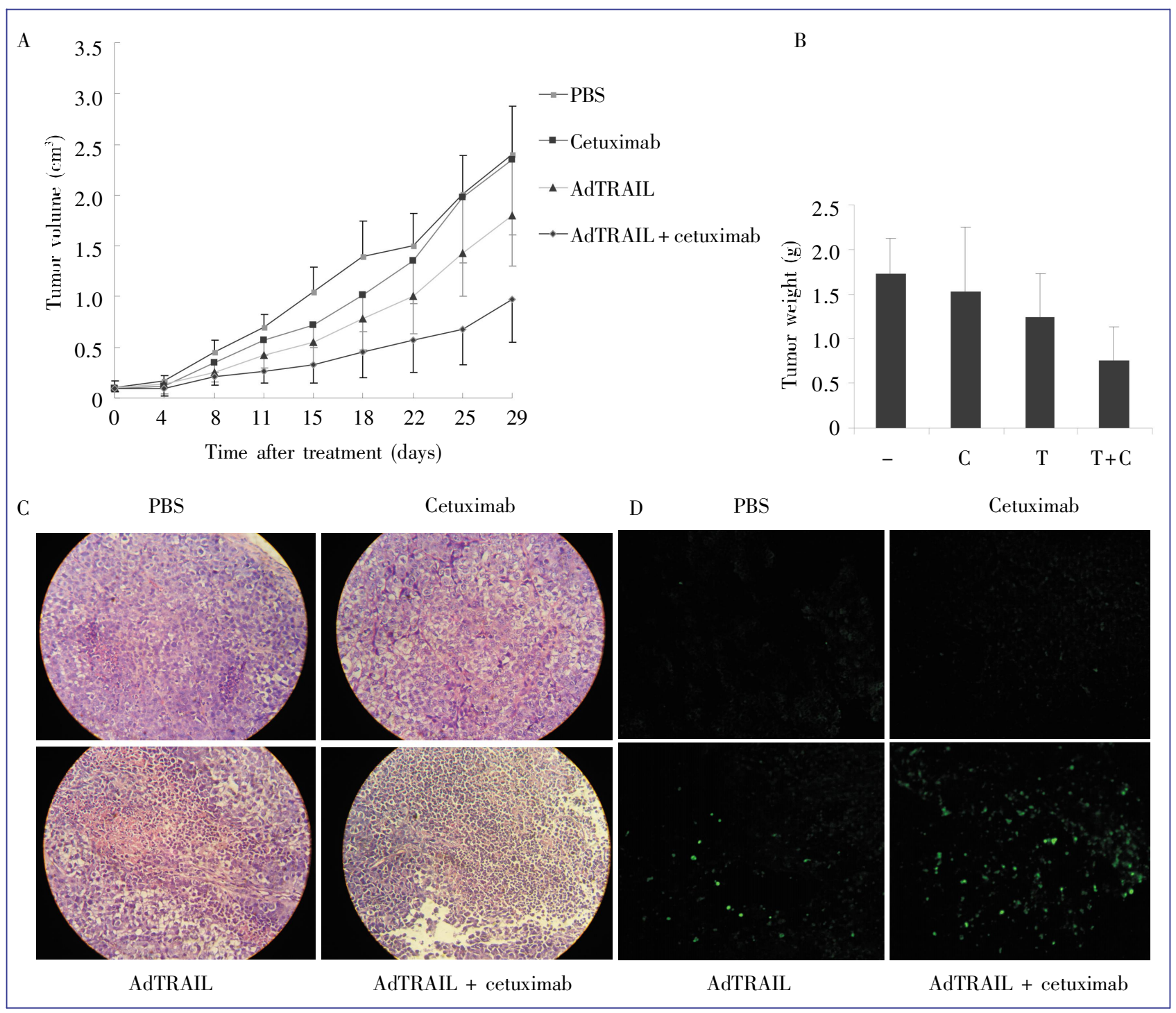

Figure 5. Effects of AdTRAIL and cetuximb on the growth of human NSCLC xenografts. The dorsal flank of female nude mice was implanted with a piece of $\mathrm{H} 460$ xenograft. Nine days later, mice were randomized and underwent indicated treatments for 21 days. Mice were killed on the 29th day after the first treatment. A, growth curve of $\mathrm{H} 460$ xenografts. All values are presented as mean \pm SD of relevant groups. ${ }^{\star}$ Compared to control treatment, combination treatment significantly inhibited the growth rate of $\mathrm{H} 460$ xenografts $(P<0.05)$. B, mean tumor weight of $\mathrm{H} 460$ xenografts (mean $\pm \mathrm{SD})$. \# Compared to control treatment, combination treatment significantly reduced the mean weight of $\mathrm{H} 460$ xenografts $(P<$ 0.05) (-, PBS; C, cetuximab; T, AdTRAIL). C, histological representative sections of xenografts were excised 29 days after treatment and stained with HE ( $\times 200)$. Tumors treated with AdTRAIL and cetuximab in combination exhibit more cell death and apoptosis. D, representative TUNEL staining of H460 NSCLC xenografts treated with the indicated agents. Combination treatment resulted in increased cell apoptosis $(\times 200)$.

AdTRAIL group $(n=8)$, and $(0.75 \pm 0.39) \mathrm{g}$ in combination treatment group $(n=9)$. The mean tumor weight was significantly lower in the combination treatment group than in control group $(P<0.05)$. Moreover, compared to control, the tumor inhibition rates were $27 \%$ in AdTRAIL group and $56 \%$ in combination treatment group. These results revealed that cetuximab enhanced the antitumor activity of AdTRAIL in vivo.
Histological examinations showed increased cell death and apoptosis in combination treatment group than in single agent group (Figure 5C). Evidence to support more tumor cell apoptosis in the xenografts after combined treatment was provided by TUNEL staining (Figure 5D). Taken together, these results suggested that cetuximab enhanced the antitumor activity of AdTRAIL in vivo by augmenting apoptosis induced by 
AdTRAIL.

Some studies showed that TRAIL could damage hepatic cells and liver function in animals ${ }^{[2]}$; however, we observed no obvious adverse effect of combination treatment with AdTRAIL and cetuximab. Histological examinations of the major organs of mice confirmed that combination treatment did no obvious pathologic damage.

\section{Discussion}

EGFR inhibitors have been important therapies for NSCLC. However, only a minority of patients with advanced NSCLC can benefit from these agents. Studies have shown that EGFR TKIs, including gefitinib and elotinib, can produce objective response and prolong progression-free survival in NSCLC patients with EGFR mutation $^{[8-10]}$. Most NSCLC patients have a wild-type EGFR, resulting in limited response to EGFR inhibitors. The three NSCLC cell lines used in our study all express wild-type EGFR ${ }^{[27]}$. In our hands, these cell lines were resistant to TKIs, which is consistent with the results of a previous study ${ }^{[27]}$. We also found that cetuximab alone had mild to moderate activity in in vitro NSCLC models, even at high concentrations. These results illustrate the need for a therapy having synergism with EGFR inhibitor in NSCLC.

TRAIL therapy is effective against many cancer types, including NSCLC; yet, in some NSCLC cell lines, the effect is limited ${ }^{[23]}$. In our study, AdTRAIL had a dose-dependent cytotoxic effect in NSCLC cell lines, and this effect became significant at high $\mathrm{MOI}$. Previous studies have often focused on enhancing the activity of AdTRAIL by increasing the transduction and transcription of TRAIL in lung cancer cells ${ }^{[28]}$. However, because normal human liver cells and certain epithelial cells are susceptible to recombinant TRAIL proteins ${ }^{[26]}$, serious concerns about the potential toxicity of high-dose TRAIL protein administered systemically have been raised. Therefore, drugs that augment AdTRAIL activity at low MOI without increasing toxicity are needed.

TRAIL induces malignant cell death or apoptosis via the extrinsic apoptotic pathway, and the EGFR pathway and extrinsic apoptosis pathway interact closely in epithelial-derived cells ${ }^{[24]}$. Some studies showed that EGF protects epithelial-derived cells from TRAIL-induced apoptosis ${ }^{[29]}$. Therefore, inhibition of the EGFR pathway may enhance TRAIL-induced apoptosis. EGFR inhibitors, including TKIs and cetuximab, can restrain the EGFR signaling pathway in NSCLC cells and provoke cell death, as demonstrated in preclinical studies and in clinical practice. These observations have fueled speculation that the combination of AdTRAIL and EGFR inhibitors may have synergistic effects in NSCLC. As shown in our in vitro study, proliferation of all three
NSCLC cell lines was inhibited when low MOI AdTRAIL was combined with EGFR inhibitors, even at low concentrations at which the inhibitors had light to mild cytotoxic effects. We selected the $\mathrm{H} 460$ cell line, which was mildly responsive to EGFR inhibitors and had obvious loss of cell viability after combination treatment, to determine whether this synergism was dependent on the enhancement of TRAIL activity. Flow cytometry analysis showed that after combination treatment, NSCLC cells were arrested in sub- $G_{1}$ phase and more apoptotic cells appeared. Reports have indicated that TRAIL-induced apoptosis requires BAX, a pro-apoptotic $\mathrm{Bcl}-2$ protein, for t-BID-mediated mitochondrial activation of caspase- ${ }^{[30]}$. Our Western blotting study showed that combination treatment caused BAX up-regulation, suggesting that the enhancement of AdTRAIL activity may underlie or contribute to the mechanisms of synergism. Furthermore, down-regulation of AKT or ERK, which are key components of the EGFR pathway, has been reported to sensitize cells to the effects of TRAIL ${ }^{[29,31]}$. Our results showed that compared to AdTRAIL alone, combination treatment caused down-regulation of $\mathrm{p}$-AKT levels but did not change p-ERK levels. These results, which are consistent with previous studies ${ }^{[29,31]}$, indicate that the AKT pathway may play a role in the synergism between AdTRAIL and EGFR inhibitors. High levels of endogenous NF-kB activity have been reported to interfere with TRAIL-induced apoptosis, and NF-kB inhibition sensitized lung cancer cells to TRAIL ${ }^{[32]}$. In our study, both combination and AdTRAIL treatments resulted in the same low NF-kB level, which indicated that NF-kB was not the main factor that contributed to this apoptosis enhancement. Therefore, we concluded that this enhancement was dependent upon reinforcement of AdTRAIL activity by down-regulation of p-AKT.

Cetuximab is an lgG1 monoclonal antibody and can induce immune response in vivo, for example, antibody-dependent cellular cytotoxicity (ADCC) and complement activation. Previous studies showed that cetuximab had potential ADCC activity against EGFR-expressing lung cancer cell lines, even those with very weak EGFR expression levels that are faintly detectable by immunohistochemistry ${ }^{[33]}$. In the present study, we found that cetuximab had mild or moderate effect on these NSCLC cell lines in vitro. H460 was reported to have moderate EGFR expression level ${ }^{[34]}$. In vitro cetuximab cannot cause ADCC or other immunity activity to inhibit $\mathrm{H} 460$ proliferation. In vivo results may be different. So we chose cetuximab for animal study and further proved this synergism. Cetuximab showed no effect on H460 cell xenografts. However, when combined with AdTRAIL, cetuximab inhibited xenograft growth significantly and increased tumor cell apoptosis. Thus, we postulated that cetuximab could augment the effect of AdTRAIL by enhancing TRAIL-induced apoptosis. 
Notably, the combination treatment had no significant effect on the weight or major organs of the mice, suggesting that combination treatment was safe and tolerable.

In summary, this pilot study provides the first evidence that EGFR pathway inhibitors, including TKIs and cetuximab, augment the antitumor effect of AdTRAIL

\section{References}

[1] Brabender J, Danenberg KD, Metzger R, et al. Epidermal growth factor receptor and HER2-neu mRNA expression in non-small cell lung cancer is correlated with survival [J]. Clin Cancer Res, 2001,7(7):1850-1855.

[2] Fontanini G, De Laurentiis M, Vignati S, et al. Evaluation of epidermal growth factor-related growth factors and receptors and of neoangiogenesis in completely resected stage I-IIIA non-small-cell lung cancer: amphiregulin and microvessel count are independent prognostic indicators of survival [J]. Clin Cancer Res, 1998,4(1):241-249.

[3] Rusch V, Klimstra D, Venkatraman E, et al. Overexpression of the epidermal growth factor receptor and its ligand transforming growth factor alpha is frequent in resectable nonsmall cell lung cancer but does not predict tumor progression [J]. Clin Cancer Res, 1997,3(4):515-522.

[4] Ritter CA, Arteaga CL. The epidermal growth factor receptortyrosine kinase: a promising therapeutic target in solid tumors [J]. Semin Oncol, 2003,30(1 Suppl 1):3-11.

[5] Pirker R, Pereira JR, Szczesna A, et al. Cetuximab plus chemotherapy in patients with advanced non-small-cell lung cancer (FLEX): an open-label randomised phase III trial [J]. Lancet, 2009,373(9674): 1525-1531.

[6] Butts CA, Bodkin D, Middleman EL, et al. Randomized phase II study of gemcitabine plus cisplatin or carboplatin, with or without cetuximab, as first-line therapy for patients with advanced or metastatic non small-cell lung cancer [J]. J Clin Oncol, 2007,25(36):5777-5784.

[7] Rosell R, Robinet G, Szczesna A, et al. Randomized phase ॥ study of cetuximab plus cisplatin/vinorelbine compared with cisplatin/vinorelbine alone as first-line therapy in EGFRexpressing advanced non-small-cell lung cancer [J]. Ann Oncol, 2008, 19(2):362-369.

[8] Fukuoka M, Yano S, Giaccone G, et al. Multi-institutional randomized phase II trial of gefitinib for previously treated patients with advanced non-small-cell lung cancer (The IDEAL 1 Trial) [J]. J Clin Oncol, 2003,21(12):2237-2246.

[9] Kris MG, Natale RB, Herbst RS, et al. Efficacy of gefitinib, an inhibitor of the epidermal growth factor receptor tyrosine kinase, in symptomatic patients with non-small cell lung cancer: a randomized trial [J]. JAMA, 2003,290 (16):21492158.

[10] Niho S, Kubota K, Goto K, et al. First-line single agent treatment with gefitinib in patients with advanced non-small-cell lung cancer: a phase II study [J]. J Clin Oncol, 2006,24(1): 64-69.

[11] Giaccone G, Herbst RS, Manegold C, et al. Gefitinib in combination with gemcitabine and cisplatin in advanced non small-cell lung cancer: a phase III trial-INTACT 1 [J]. J Clin Oncol, 2004,22(5):777-784.

[12] Herbst RS, Giaccone G, Schiller JH, et al. Gefitinib in combination with paclitaxel and carboplatin in advanced non small-cell lung cancer: a phase III trial-INTACT 2 [J]. J Clin Oncol, 2004,22(5):785-794. in NSCLC cell lines. Furthermore, inhibiting AKT is important for apoptosis enhancement induced by combination treatment.

Received: 2011-03-17; revised: 2011-08-05; accepted: 2011-08-18.
[13] Herbst RS, Prager D, Hermann R, et al. TRIBUTE: a phase III trial of erlotinib hydrochloride (OSI-774) combined with carboplatin and paclitaxel chemotherapy in advanced nonsmall-cell lung cancer [J]. J Clin Oncol, 2005,23 (25):58925899 .

[14] Gatzemeier U, Pluzanska A, Szczesna A, et al. Phase III study of erlotinib in combination with cisplatin and gemcitabine in advanced non-small cell lung cancer: the Tarceva Lung Cancer Investigation trial [J]. J Clin Oncol, 2007,25(12):1545-1552.

[15] Suliman A, Lam A, Datta R, et al. Intracellular mechanisms of TRAIL: apoptosis through mitochondrial-dependent and -independent pathways [J]. Oncogene, 2001,20 (17):2122 2133.

[16] Wiley SR, Schooley K, Smolak PJ, et al. Identification and characterization of a new member of the TNF family that induces apoptosis[J]. Immunity, 1995,3(6):673-682.

[17] Ashkenazi A, Pai RC, Fong S, et al. Safety and antitumor activity of recombinant soluble Apo2 ligand [J]. J Clin Invest, 1999, 104(2): 155-162.

[18] MacFarlane M. TRAIL-induced signalling and apoptosis [J]. Toxicol Lett, 2003,139(2-3):89-97.

[19] Wang S, El-Deiry WS. TRAIL and apoptosis induction by TNFfamily death receptors [J]. Oncogene, 2003,22(53):8628-8633.

[20] Lee J, Hampl M, Albert P, et al. Antitumor activity and prolonged expression fromaTRAIL-expressing adenoviral vector [J]. Neoplasia, 2002,4(4):312-323.

[21] Lin $T$, Huang $X, G u J$, Zhang $L$, et al. Long-term tumor-free survival from treatment with the GFP-TRAlL fusion gene expressed from the hTERT promoter in breast cancer cells [J]. Oncogene, 2002,21(52):8020-8028.

[22] Katz MH, Spivack DE, Takimoto $S$, et al. Gene therapy of pancreatic cancer with green fluorescent protein and tumor necrosis factor-related apoptosis-inducing ligand fusion gene expression driven by a human telomerase reverse transcriptase promoter [J]. Ann Surg Oncol, 2003,10(7):762-772.

[23] Voortman J, Resende TP, Abou El Hassan MA, et al. TRAIL therapy in non-small cell lung cancer cells: sensitization to death receptor-mediated apoptosis by proteasome inhibitor bortezomib [J]. Mol Cancer Ther, 2007,6(7):2103-2112.

[24] Gibson SB. Epidermal growth factor and trail interactions in epithelial-derived cells [J]. Vitam Horm, 2004,67:207-227.

[25] Kagawa S, He C, Gu J, et al. Antitumor activity and bystander effects of the tumor necrosis factor-related apoptosis-inducing ligand (TRAIL) gene [J]. Cancer Res, 2001,61(8):3330-3338.

[26] Jo M, Kim TH, Seol DW, et al. Apoptosis induced in normal human hepatocytes by tumor necrosis factor-related apoptosisinducing ligand [J]. Nat Med, 2000,6(5): 564-567.

[27] Coldren CD, Helfrich BA, Witta SE, et al. Baseline gene expression predicts sensitivity to gefitinib in non-small cell lung cancer cell lines [J]. Mol Cancer Res, 2006,4(8):521-528.

[28] Kim DR, Park MY, Lee CS, et al. Combination of vorinostat and adenovirus-TRAIL exhibitsa synergistic antitumor effect by increasing transduction and transcription of TRAIL in lung 
cancer cells [J]. Cancer Gene Ther, 2011,18(7):467-477.

[29] Gibson EM, Henson ES, Haney N, et al. Epidermal growth factor protects epithelial-derived cells from tumor necrosis factor-related apoptosis-inducing ligand-induced apoptosis by inhibiting cytochrome c release [J]. Cancer Res, 2002,62(2): 488-496.

[30] Ravi R, Bedi A. Requirement of BAX for TRAIL/Apo2L-induced apoptosis of colorectal cancers: synergism with sulindacmediated inhibition of Bcl-x (L) [J]. Cancer Res, 2002,62(6): 1583-1587.

[31] Park SY, Seol DW. Regulation of Akt by EGF-R inhibitors, a possible mechanism of EGFR-R inhibitor-enhanced TRAILinduced apoptosis [J]. Biochem Biophys Res Commun,
2002,295(2):515-518

[32] Aydin C, Sanlioglu AD, Bisgin A, et al. NF-kB targeting by way of IKK inhibition sensitizes lung cancer cells to adenovirus delivery of TRAIL [J]. BMC Cancer, 2010,10:584.

[33] Kurai J, Chikumi H, Hashimoto K, et al. Antibody-dependent cellular cytotoxicity mediated by cetuximab against lung cancer cell lines [J]. Clin Cancer Res, 2007,13(5):1552-1561.

[34] Van Schaeybroeck S, Kyula J, Kelly DM, et al. Chemotherapyinduced epidermal growth factor receptor activation determines response to combined gefitinib/chemotherapy treatment in nonsmall cell lung cancer cells [J]. Mol Cancer Ther, 2006,5(5): 1154-1165.
Submit your next manuscript to Chinese Journal of Cancer and take full advantage of:

- Open access

- No charge to authors

- Quickly published

- Thorough peer review

- Professionally edited

- No space constraints

- Indexed by PubMed, CA, and Google Scholar

Submit your manuscript at

www.cjcsysu.com 